\title{
Assessment Tools Preferred by the Undergraduate Clinical Medical Students: A Study in National Defense University of Malaysia
}

\author{
S.S. Ahmed and S.C. Reddy
}

\section{ABSTRACT}

Background: Assessment in medical education is necessary to determine the students' competency as well as other attributes.

Objectives: The aim of this study was to determine the methods of assessment at the end of rotational posting examination and final professional examination at the end of the course, preferred by the undergraduate clinical medical students.

Methods: This study was conducted on 89 medical students belonging to $4^{\text {th }}$ and $5^{\text {th }}$ year of the Faculty of Medicine, National Defense University of Malaysia (NDUM).

Results: In this study 46 students were males $(51.7 \%)$ and $43(48.3 \%)$ females; the mean age was 23.5 years. Among the multiple-choice questions (MCQ), a combination that contained multiple true-false and single best answer, was preferred by $55.1 \%$ of the students. Among the essay type of written assessments "problem management patient (PMP)" questions were preferred most $\mathbf{( 4 6 . 1 \% )}$ followed by modified essay questions (MEQ), short essay questions (SEQ), and key feature questions (KFQ). Among the assessments by long case, conventional long case, which had been practiced during the course, was preferred by $67.4 \%$ of the students at the end of the medical program rather than the modified or observed long case examinations. While assessment by short case, running commentary on what was being examined, was preferred by $47.2 \%$ of students, followed by no commentary $(38.2 \%)$ and not sure by the rest. $56.2 \%$ of students would prefer procedure OSCEs on mannequins and the rest won't. Picture quizzes were highly preferred $(89.9 \%)$.

Conclusion: At the end of the medical program the clinical medical students prefer those methods which have already been practiced by them during the academic course. For the best achievement, every tool of assessments used in the medical curriculum should be made clear to the students and the students should practice those before they proceed for final evaluation.

Keywords: Assessment methods, clinical examinations, medical school, undergraduate medical education, written examinations.

\section{INTRODUCTION}

The ultimate aim of undergraduate medical education is to produce a competent, safe and caring doctor. Assessment in medical education is necessary to determine the students' competency as well as other attributes. Millers has described four steps of assessment in his pyramid beginning from the base as "knows" (test of factual recall of knowledge), "knows how" (test of knowledge application in problem solving and decision making), "shows" (test of clinical competency) and "does" (test of performance at the workplace) [1]. The knowledge of medical science and its application is assessed by close and open-ended written examinations whereas
Published Online: August 23, 2021

ISSN: $2736-5476$

DOI: $10.24018 /$ ejclinicmed.2021.2.4.112

\section{S. S. Ahmed}

Department of Medicine, National Defense University of Malaysia, Kuala Lumpur, Malaysia.

Department of Medicine, Royal College of Surgeons in Ireland and University College Dublin, Malaysia Campus, Penang, Malaysia.

S. C. Reddy*

Department of Ophthalmology, National Defense University of Malaysia, Kuala Lumpur, Malaysia.

(e-mail: profscreddy ${ }^{\circledR}$ gmail.com)

*Corresponding Author clinical skills of dealing patients and other competencies are assessed by clinical examination. The formats of written examinations are multiple-choice questions (MCQ) and NonMCQ, the latter commonly are open ended questions in the form of modified essay questions (MEQ), short answer questions (SAQ) and problem management patient (PMP). The formats of clinical assessments are long case, short case and observed structured clinical examination (OSCE). The OSCE may be interactive (examples are the student interacts with a standardized patient or performs a specified task, which are carried out in front of the assessor) and noninteractive (assessors do not remain standing beside the student for observation and judgement). Example of written 
closed questions or MCQs are multiple true-false (MTF), single best answer (SBA) and extended matching questions (EMQ); other examples of open-ended questions include short essay questions (SEQ) and key feature approach questions (KFQ) [2].

Each medical institution has a clear course contents and learning outcomes in their medical curriculum guidebooks which are provided to the students. A blueprint (table of specifications) specifies in detail what content needs to be covered in the assessment and ensures the quality of an assessment as well as the validity of the results [3]. The three learning domains described by Bloom et al. [4] in 1956, that consist of cognitive (e.g., knowledge), psychomotor (e.g., skills), and affective (e.g., attitudes) are assessed by various tools. Historically, the qualities of various tools for assessment used were validity and reliability; in 1996, van der Vleuten expanded the list to include acceptability, cost effectiveness and educational impact [5]. Finally, the criteria were further reaffirmed and added to in a consensus statement of the 2010 Ottawa Conference [2], [6]. However, there is no single tool that possesses all the qualities of a good assessment or that assess other characteristics such as the learning domains, hence the necessity of multiple modalities of assessment. This principle also reflects the students' characteristics of the combination of various learning styles for an effective learning that includes reading/writing, hearing, seeing (observing), and practicing of skills [7].

Currently in various medical institutions, there is a little variation in the assessment types and formats; however, the fundamentals remain the same. During the whole tenure of a medical course, 2 types of assessments are usually carried out. One of the assessments is taken while the course of medical education is running on and is traditionally known as formative assessment. The purpose of formative assessment is to see the efficacy of learning, to provide feedback and to create methods of improvement of learnings and teachings which is an integral part of education. Mini clinical evaluation exercises (MiniCEX), and direct observation of procedural skills (DOPS) are the examples of formative assessment for the housemen. The objective structured long case examination records (OSLER) are often carried out for the undergraduates beside MiniCEX, logbook and portfolio assessments. The second type of assessment is taken at the end of the whole course known as the summative assessment that certifies the student with grades to become a graduate; the passing criteria are based on examination standards. Standard setting is the process of demarcating the level of knowledge and skill indicating proficiency and identifying a score on the examination that determines pass or fail of a candidate [8].

During the course, continuous assessments are often carried out at the end of each discipline posting /rotations. The marks of the continuous assessments are carried to the summative assessment; therefore, summative assessments are usually cumulative. The continuous assessment may include written and clinical examinations, case write up assignments, attitude assessment, portfolio review as well as logbook review that shows the recording of number of activities such as clinical cases seen in the wards/clinics, procedures observed in the wards/clinics, number of deliveries conducted in the labor room, number of operations seen in the operation

\section{theatre, etc.}

In this paper we describe the common tools for assessment that were preferred by the clinical medical students at one of the institutions in Malaysia during their period of educational learning (at the end of rotational postings) as well as at the end of the medical course. Assessments are an essential part of the teaching and learning process; its evaluation has an impact on the quality of education and student's achievement. Assessment forces learning and teachings in medical education. The students may have some fascination in choosing the assessment methods. Whatever assessment methods are carried out by the institution, it must be conveyed clearly to the students during their learning and teaching periods and before proceeding to the examinations to make the students very familiar with the tools. There is a great sparsity of studies on the preference of tools of assessment by undergraduate clinical medical (specially $4^{\text {th }}$ and $5^{\text {th }}$ year) students in a medical institute. Therefore, this study was conducted to investigate the preferred methods of assessment by the undergraduate clinical medical students in the faculty of medicine of National Defense University of Malaysia (NDUM).

\section{MATERIALS AND METHODS}

This study was conducted among 89 medical students of 4th and 5th year of the Faculty of Medicine and Defense Health, NDUM, from $1^{\text {st }}$ November 2019 to $31^{\text {st }}$ July 2020. Kuala Lumpur. This is a prospective quantitative questionnaire study, and a single preferred answer was obtained through closed ended questions with multiple answer options. The medical program is for a period of 5years; initial 2 years is a pre-clinical program. Once the student qualifies, only then he/she will proceed for a clinical course of 3-years duration. After qualifying the final professional examination at the end of 5th year, they are awarded a Doctor of Medicine (MD) degree. The study was conducted in one of the clinical teaching premises of our university (Hospital Angkatan Tentera Tuanku Mizan (Tuanku Mizan Military Hospital), Kuala Lumpur).

Inclusion criteria were (i) undergraduate medical students in 4th and 5th year, placed in clinical rotations and (ii) supplementary candidate of final professional examination. Exclusion criteria were (i) the students unwilling to be included in the study and (ii) incomplete/ inadequate response to the questionnaire.

The respondents were informed that their identity will not be revealed to anyone and the confidentiality of the participants will be maintained. A structured data collection questionnaire was prepared and validated. Ten questions were designed to cover the assessment methods of theory (4 questions) and clinical examination (4 questions) and on compulsory pass in the clinical examination (2 questions). The questions were constructed in a simple way so that it was easy to understand, and every question could be answered.

This study was approved by the research and ethics committee of our university. Data collection was done by both authors. The students were free either or not to participate in the study. After a brief and clear explanation of the purpose and procedure of the study, the questionnaires were distributed to the students and written consent was 
taken. Students responded anonymously to the study questionnaire. All participants were asked to tick one of their appropriate responses against the questions. Completed questionnaires were collected. The information that was collected in the structured data collection form were entered and analyzed in Statistical Package for Social Sciences (SPSS) version-20.

\section{RESULTS}

This study was carried out among 89 students of the undergraduate medical program of the NDUM who were in their advanced clinical postings. Out of 89 students, 46 were males $(51.7 \%)$ and $43(48.3 \%)$ were females. The mean age was found to be 23.5 years (range 23-27 years).

Table I illustrates the students' response for their preferred methods of assessment. Regarding MCQ, a combination of
MTF and single best answer (both mixed) was preferred by $55.1 \%$ of students. Majority of students $(46.1 \%)$ preferred PMP type of theory questions. In the assessment, picture quizzes were preferred in $89.9 \%$ of students. Regarding long case examination, a traditional method of one-hour clerking followed by presentation and examination by the examiner for 20-30 minutes was preferred by $67.4 \%$ of students. During short case examination, giving running commentary what is being examined, was preferred by $47.2 \%$. Regarding OSCE, non-interactive was preferred by $50.6 \%$ of students. Majority of students $(56.2 \%)$ preferred procedure OSCE on a mannequin. At the end of posting/rotation (EOP) assessment, compulsory pass of clinical examination (without contribution from other components) was preferred by $43.8 \%$ of students. If failed in the clinical examination at the EOP assessment, then clinical examination alone was preferred by majority $(55.1 \%)$ of students.

TABLE I: STUdENTS’ RESPONSES For QUESTIONS On ASSESSMENT METHODS (N=89)

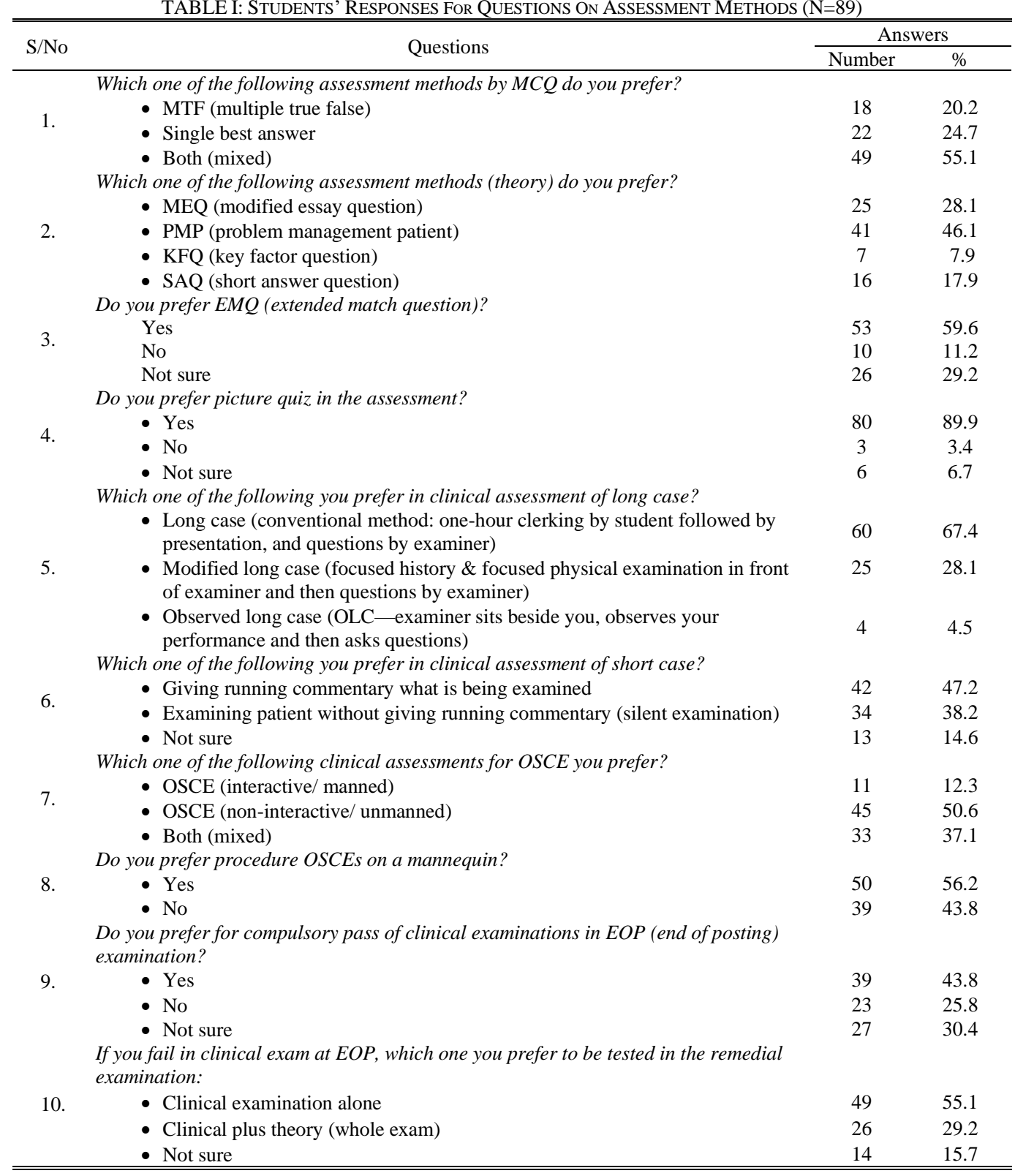

\section{DISCUSSION}

The purpose of this study was to find out the medical 
students' preferred methods of assessment during their clinical placement (end of posting examination) and at the end of medical course (final professional examination).

During formative as well as summative assessments common methods of assessment of knowledge and its application that were practiced by the faculty of medicine in NDUM were MCQs and non-MCQ written papers (MEQ, PMP, KFQ, and SAQ). These papers contained mostly clinical scenarios with problems and patient management tasks, data analysis and clinical reasoning. In this study, among the MCQs, a combination that contained MTF and SBA (single best answer) type MCQ, was preferred by $55.1 \%$ of students followed by preference of single best answer (24.7\%) and MTF answers (20.2\%). Among the other (nonMCQ) written assessments, PMP was preferred by $46.1 \%$ of students followed by MEQ (28.1\%), SAQ (17.9\%), and KFQ (7.9\%). In the assessments, picture quizzes were highly preferred by the clinical medical students $(89.9 \%)$.

In multiple true /false answers (MTF) question, a statement is given as a stem and five answers are written below that. The student has to write each answer true or false in relation to the statement given. The short answer question (SAQ) is an open ended, semi-structured question format. A structured predetermined marking scheme improves objectivity. The questions can incorporate clinical scenarios. SAQs have a better content coverage as compared to long essay question. Key factor question is a clinical scenario-based paper and pencil test. A description of the problem is followed by a limited number of questions that focus on critical, challenging actions or decisions. It has higher content validity with proper blueprinting [9].

Traditional MTF questions are useful where it is important to assess factual recall of essential knowledge, for example, in management protocols for emergency situations. However, this style of question does not allow facts to be placed in context or lend it to testing the application of knowledge and problem solving that is so essential in clinical practice. This is where SBAs can be invaluable; they allow the candidate to demonstrate that they 'know how' rather than simply 'know', and this is a fundamental principle of the assessment of clinical skill" [10].

Almothafar and Chee from Malaysia studied the performance of final year medical students in the end of the surgical posting examination and reported that the students appear to be weaker in their MCQs, demonstrating a decline in theoretical knowledge; there is a poor positive correlation between theoretical knowledge, clinical skills and/or continuous assessments, demonstrating the importance of emphasis in these three areas amongst medical students [11].

The chance of selecting the correct choice is $20 \%$ in the SBA question type with a stem and five options (4 distracters) while the odds of guessing the correct answer is obviously $50 \%$ to each sub-item of a five-item MTF question form. Hence, there is strong possibility that examinees can score higher marks in MTF format than the SBA format [12].

Multiple choice questions alone or in conjunction with other test instruments are extensively used in high-stakes examination in medical institutions. Amongst numerous genres of MCQ style of exam with five options, it seems that examinees have greater chance to get correct answers for multiple true-false (MTF) than single best answer (SBA).
Simbak et al. [13] from Malaysia examined students' performance between MTF and SBA evaluation techniques and correlated them with other assessment outcomes. The results pointed out that the students got higher marks in SBA questions than MTF quiz. SBA test results were found to be well correlated with OSCE marks, long case examination and modified essay question (MEQ). The findings of this study encouraged the use of SBA quiz in place of MTF test format for examinations. However, the diverse teaching techniques, variation of competency of lecturers, dissimilar marking systems and different question difficulty indexes between these two different subjects may contribute unequal scores and can lead to bias in comparison of MTF and one best answer formats [13].

Farooqui et al. [14] from Pakistan conducted a study to see the correlation between MCQs and SAQs in the end-ofclerkship examinations in different subjects for 481 final-year medical students over a period of five years and found a statistically significant overall correlation between students' performance on MCQ and SAQ in all the four major subjects (surgery, medicine, pediatrics and obstetrics \& gynecology).

A well-structured MCQ should be considered as a preferable choice in undergraduate medical education as different levels of Bloom's taxonomy can be assessed by this assessment format and its use for assessing only lower ordered thinking i.e. recall of knowledge, is not very convincing. The literature search also supports for using multiple choice questions for assessment of higher ordered thinking [15].

The common tools for assessment of clinical competency used by the medical faculty of the university were conventional long cases and short cases (on real patients), as well as OSCEs. During the formative assessments a part or all the tools were used according to the suitability of the subject module; while in the summative assessment, all the tools were utilized as different disciplines participated together in the final examination. In this study, overall assessment by conventional long case was preferred by most of the students $(67.4 \%)$, and the rest preferred either observed $(4.5 \%)$ or modified long case $(28.1 \%)$. In the final examination at the end of the course, there was a trend by the university of using the observed/modified long case, but in this study, the students have shown fewer preferences to those ways of clinical assessments. One of the reasons might be the lack of practice of those methods during formative/ continuous assessments in the course.

Therefore, it appears that, at the end during summative assessment, the students prefer those methods of assessments which have already been practiced by them in their formative and continuous evaluations. This indicates that whatever methods and tools of assessments used during the final evaluation, the students need to practice them during the course especially at their formative and continuous assessments. While assessment of clinical competency of the physical examination skills by short case examinations, running commentary on what is being examined, was preferred by $47.2 \%$ of students, silent examination without giving running commentary by $38.2 \%$ and not sure by $14.6 \%$ of students.

Long case may provide a unique opportunity to test the physician's tasks and interaction with a real patient. It has 
poor content validity, is less reliable and lacks consistency. Short case involves use of three to four non-standardized real patients with one to two examiners. It provides opportunity for assessment with real patients and allows greater sampling than single long case. OSCE consists of multiple stations where each candidate is asked to perform a defined task such as taking a focused history or performing a focused clinical examination of a particular system. A standardized marking scheme specific for each case is used. It is an effective alternative to unstructured short cases [9].

Mashi and Benson from UK studied third year medical students undertaking musculoskeletal attachment, through a questionnaire whether students were in favor of the long case as a formative assessment tool, reported that the long case can be a useful tool for formative assessment as well as a rich source of learning for medical students [16].

To determine the various clinical skills, a series of OSCEs that were practiced by the medical faculty in NDUM during the summative assessments were grouped into (i) interactive (manned) stations which include history taking, clinical examination, communication skills, certain diagnostic or therapeutic procedures, instruments usage, and counselling using real or standardized simulated patients or mannequins; and (ii) non-interactive (un-manned) stations which include data analysis, medical equipment usage, evaluating ECGs, XRays and blood investigations interpretation, spot diagnosis photos, etc. In the interactive OSCE, the examiner observes the performance of a student; the examiner remains close within the OSCE station and scores in the standardized checklist; and interacts with the student at the end by asking few questions about the knowledge of the OSCE topic. In unmanned OSCE stations, the examiners do not remain standing beside the student within the station, and structured markings are done at the end of the examination.

In this study, the non-interactive OSCEs were preferred by the most students $(50.6 \%)$ followed by the mixed ones $(37.1 \%)$ that consisted of interactive and non-interactive ones; OSCEs conducted only with the presence of an examiner was least preferred $(12.3 \%)$. One reason for not preferring interactive OSCEs might be inadequate exposures and practice during formative/continuous assessments conducted by the various disciplines; and the other could be too long gap (12 to 22 months) between the specialties posting in year 4 and the final professional examination at the end of year 5. In manned OSCE, $56.2 \%$ of students would prefer procedure OSCEs on mannequin, whereas $43.8 \%$ won't.

OSCEs remain a beneficial tool for learning, revision and widely used to assess the clinical performance of medical students in almost all medical students in the world. Robinson et al. [17] from UK conducted simulated mock OSCE for final year medical students over a period of two years and demonstrated that they significantly improve medical student confidence and significantly reduce anxiety related summative examinations.

Foud et al. [18] from Egypt investigated perception, among the clinical year students and staff, of OSCE as a clinical assessment tool during their clinical years through different questionnaires. They reported that OSCE is a valuable practical learning experience and most of the students agreed that OSCE helped them to identify gaps in their clinical knowledge. There were different causes of stress and dissatisfaction among students regarding the OSCE exam, including a shortage of time for stations to complete the requested task and no feedback received from the staff in some stations after the end of the examination. Eighty eight percent of the students agreed that OSCE should remain as a form of performance assessment, and $83 \%$ of them agreed that OSCE was a valuable practical exam and provided them a great learning experience. Approximately $80.4 \%$ of the staff emphasized that faculty members need specific training to achieve more valid and reliable results when using OSCE as an assessment tool, and $76.5 \%$ of them agreed that using OSCE reduced the bias in clinical assessment [18].

There was a proposal by our faculty of medicine in NDUM to introduce compulsory pass of the clinical examination at the end of posting after each rotation, and also in the remedial examination thereafter. Therefore, the last two questions were given for the response of students on this point. In formative assessments that consist mainly of written and clinical examinations, compulsory pass of the clinical examination component, was agreed by $43.8 \%$ of the students while $25.8 \%$ of the students did not agree, the rest were unsure about it. If failed, then only clinical examination alone to repeat during remedial examination instead of the whole examination (theory and clinical) was preferred by most of the students $(55.1 \%)$. This indicates that there is a trend among the students to improve their standards of clinical competency more when compared with the theoretical component.

Whatever tools of assessment are used during the summative assessment in medical education, the students need to practice those methods of assessment during the course in their clinical placement as well as during their formative assessment so that these methods become acceptable to the students and overall better performances are achieved at the end. The results of our study suggest that the students need more attention in the components of the assessment modalities which were not preferred much by them; and the teaching methods should be tailored to achieve this.

In this study, the most students choose to be declared themselves fail if they fail in the clinical examinations during continuous assessment even, they pass overall from contributions made by other components (e.g., written examinations and other elements of continuous assessment). In such case most of them prefer to repeat the assessment with the clinical examination component only; the faculty would repeat both theory and clinical examinations. This may reflect the trend in the medical students to develop satisfactory clinical competency before appearing the final examination. In one study to determine the preferred teaching methods, the bedside teaching was preferred most by the undergraduate clinical medical students, reflecting the trend in developing clinical competency in learning history taking, physical examination and other skills that they feel more helpful in the final assessment [19].

\section{CONCLUSION}

At the end of the medical program when summative assessments are carried out, the clinical medical students 
prefer those methods which have already been practiced by them in their formative evaluations. For the best achievement, every tool of assessment used in the medical curriculum should be made clear to the students and the students should practice those before they proceed for evaluation in the examination.

\section{LIMITATIONS OF THE STUDY}

The study was conducted in a part of the clinical medical students of one medical institution at Kuala Lumpur. Hence, the findings of this study do not reflect the actual preference of assessment methods of the clinical students of all the medical institutions of the country. The other limitation is a small sample size of the students.

\section{FUNDING}

Both authors declare no financial support.

\section{CONFLICT OF INTEREST}

Both authors declare no conflict of interest.

\section{ACKNOWLEDGMENT}

We are grateful to NDUM and Dean, Faculty of Medicine and Defense Health, for giving us the opportunity for conducting the study. We are thankful to the staff of the faculty for helping us in using the stationeries, papers, printer machines, etc. and for assisting to conduct the study. Lastly, we also extend our sincere thanks to all the students who participated and provided their honest responses in this study.

\section{REFERENCES}

[1] G. E. Miller, "The assessment of clinical skills/competence/ performance." Acad Med, 65:563-67, 1990.

[2] J. A. Dent, R. M. Harden, D. Hunt, "A Practical Guide for Medical Teachers.” Fifth Edition. Elsevier Ltd, 2017.

[3] S. M. Downing, T. M. Haladyna, editors: Handbook of Test Development, Mahwah, NJ, Erlbaum, 2006.

[4] B. S. Bloom, M. D. Englehart, E. J. Furst, et al. "Taxonomy of educational objectives, the classification of educational goals." Handbook I: Cognitive domain. 1st Ed. New York, NY: Longmans, Green, and Co. p 206, 1956.

[5] C. Van der Vleuten "The assessment of professional competence: Developments, research and practical implications." Adv Health Sci Educ.1:41 67, 1996.

[6] J. Norcini, B. Anderson, V. Bollela et al "Criteria for good assessment: Consensus statement and recommendations" Ottawa 2010 Conference, Medical Teacher, 33:206-14, 2011.

[7] S. S. Ahmed, S. G. Reddy, "Preferred Learning Style and Study Characteristics: A Study Among Undergraduate Clinical Medical Students of National Defense University of Malaysia." Journal of Advances in Medicine and Medical Research, 32(19):51-60, 2020.

[8] J. J. Norcini "Setting standards on educational tests." Medical Education, 37: 464-9, 2003.

[9] S. M. Tabish, "Assessment Methods in Medical Education." Int J Health Sci (Qassim), 2(2):3-7, 2008.

[10] L. Brennan, "Single best answer MCQs." RCoA Bulletin, 57:39-41, 2009.

[11] B. Almothafar, F. T. Chee, "Performance of the final year medical students in Universiti Malaysia Sabah in the end surgical senior posting (SSP) examination." JUMMEC, 14(2):1-6, 2011.

[12] M. Chandratilake, M. Davis, G. Ponnamperuma, "Assessment of medical knowledge: the pros and cons of using true/false multiplechoice questions." Natl Med J India, 24(4):225-8, 2011.
[13] N. Simbak, M. M. T. Aung, S. Ismail, N. Mat Jusoh, T. I. Ali, W. A. K. Yasin, M. Zaque, H. M. A. Rebuan, "Comparative Study of Different Formats of MCQs: Multiple True- False and Single Best Answer Test Formats, in a New Medical School of Malaysia." Int Med Journal, 21(6):562- 6, 2014.

[14] F. Farooqui, N. Saeed, S. Aaraj, S. Ma, M. A. Amir, "Comparison Between Written Assessment Methods: Multiple-choice and Short Answer Questions in End-of-clerkship Examinations for Final Year Medical Students." Cureus, 10(12): e3773, 2018.

[15] A. Javeed, "Assessment of Higher Ordered Thinking in Medical Education: Multiple Choice Questions and Modifi ed Essay Questions." MedEdPublish, 7(2), 2018.

[16] C. S. Masih, C. Benson, "The Long Case as a Formative Assessment Tool - Views of Medical Students.” Ulster Med J, 88(2):124-7, 2019.

[17] P. Robinson, L. Morton, H. Haran, R. Manton, "Mock OSCEs improve medical students' confidence and reduce anxiety related to summative examinations." Education in Medicine Journal, 9(2):41-45, 2017.

[18] S. Fouad, E. Gouda, A. Abdel Nasser, D. Kamal, "Perception of students, staff and simulated patients towards objective structured clinical examination (OSCE)." Education in Medicine Journal, 11(2):27-42, 2019.

[19] S. S. Ahmed, S. C. Reddy, "Clinical Medical Students' Preferred Teaching Methods: A Study at the National Defense University of Malaysia." 3(2):74-8, 2021. 